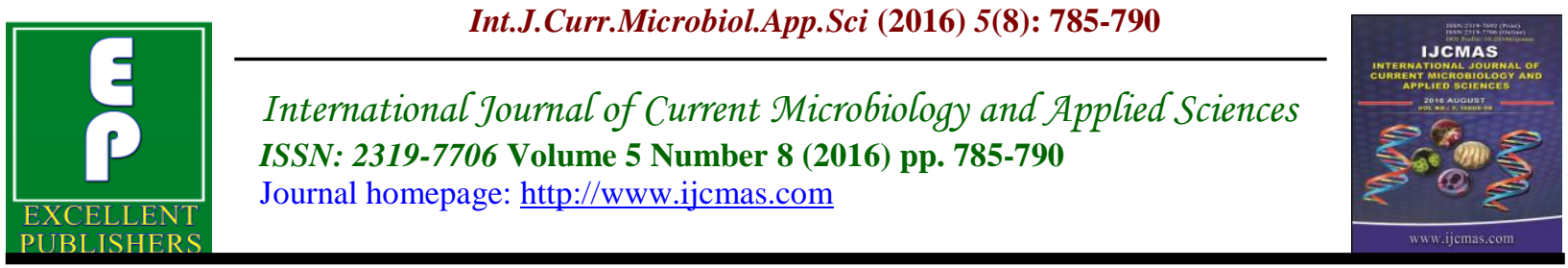

Original Research Article

http://dx.doi.org/10.20546/ijcmas.2016.508.087

\title{
Microbiological Profile of Neonatal Sepsis with Special Reference to Umbilical Stump Infections
}

\author{
S. Rajesh, R. Vidhya Rani* and T. Sundararajan \\ Department of Microbiology, Govt. Medical College, Salem Tamilnadu, Pin-636030, India \\ *Corresponding author
}

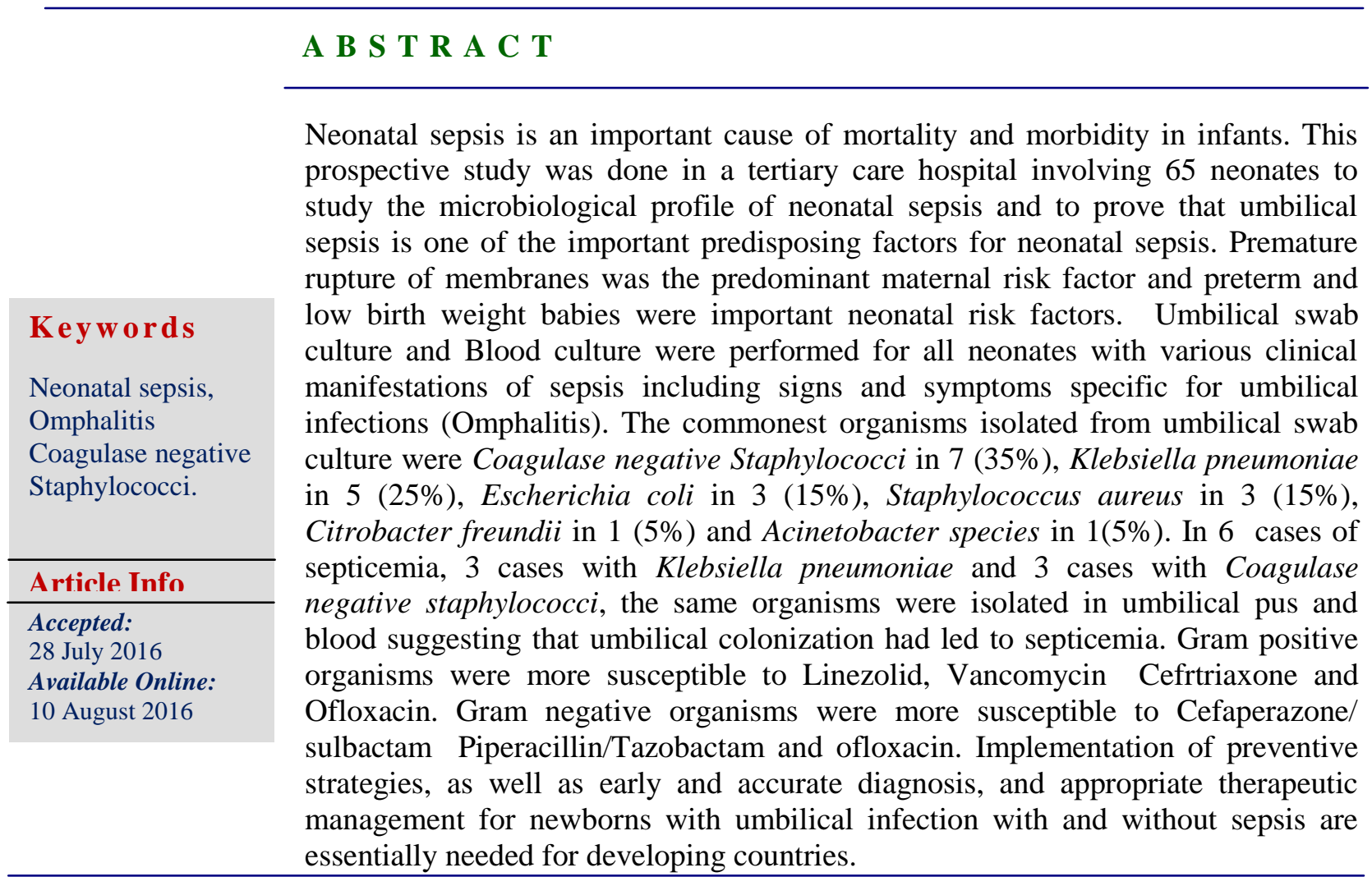

\section{Introduction}

Neonatal sepsis is defined as a clinical syndrome of bacteremia with systemic signs and symptoms of infections within first four weeks of life.. Neonatal sepsis can be classified into two major categories as early onset and late onset depending on the onset of symptoms.
Early onset sepsis (EOS) occurs within the first week of life. The source of infection is generally the maternal genital tract. Some maternal/perinatal conditions have been associated with an increased risk of EOS (Mukhopadhyay et al., 2009). Late onset sepsis (LOS) occurs in after the first week 
until the end of the neonatal period. The source of infection in LOS is either hospital-acquired or community acquired and neonates usually present with septicemia, pneumonia or meningitis (Camacho-Gonzalez et al., 2013). Neonatal infections currently cause about 1.6 million deaths annually in developing countries (Liu et al., 2012).

Omphalitis or umbilical infections are common among newborns in developing countries and may predispose to lifethreatening neonatal sepsis (Mullany et al., 2009).

There is an uprise in the incidence in developing countries because the neonatal population includes mainly term babies looked after in high dependency units, with scarce supportive and monitoring equipment, overcrowding, poor staffing levels, and difficulty in providing even basic supportive treatment (Fikree et al., 2005). Unsanitary delivery practices, poor hand washing, unclean delivery surfaces, and unsterile cord cutting and tying may contribute to umbilical stump infection (Bahl et al., 2009). Coagulase-negative staphylococci are the major pathogen involved in late onset neonatal sepsis., particularly in infants born at a lower gestational age.

\section{Aims and Objectives}

1. To study the microbiological profile and to know the antimicrobial susceptibility pattern for early therapeutic intervention and decrease the Infant Mortality Rate.

2. To establish the importance of umbilical swab culture in diagnosing neonatal sepsis and incorporating it as one of the routine screening methods for the detection of neonatal sepsis
3. To prove that surface colonizing bacteria of umbilical stump are important in neonatal sepsis.

\section{Materials and Methods}

This is a prospective study done for two months from March- April 2016 at Government Mohankumaramangalam Medical College Hospital, Salem. The study group involved newborn babies upto one month of age with suspected neonatal sepsis.

Study procedure: The study is carried out after the clearance and approval by the Institutional Ethical Committee (IEC).

\section{Inclusion Criteria}

Newborn babies of age group upto one month that have risk of neonatal sepsis like

\section{Maternal factors}

Premature rupture of membranes, Prolonged labour, Chorioamnionitis Urinary Tract Infections Multiple vaginal examinations $\mathrm{H} / \mathrm{O}$ intrauterine deaths or spontaneous abortions

\section{Newborn factors}

$\mathrm{H} / \mathrm{O}$ any indigenous practises Preterm or prematurity Low apgar score Poor umbilical care

Use of invasive procedures like ET tube, chest drain or IV cannula with signs of neonatal sepsis like lethargy, refusal to suck, poor cry, not arousable, abdominal distention, diarrhea, vomiting, hypothermia, poor prefusion, fever, blank look, high pitched cry, chest retractions, grunt gasping, seizures, excessive crying shock, bleeding, renal failure, bulging fontanel, neck retraction, 
Umbilical swab sample was collected from these babies which show signs of omphalitis like periumbilical erythema umbilical discharge (bloody, serosanguinous, purulent). A sterile swab wetted with normal saline is used to collect pus sample from around the umbilical stump. The wound is cleansed with normal saline and the swab is collected. Both pus and wound swabs were collected. 2 swabs one each for direct gram staining and aerobic bacterial culture were collected.

Blood samples were collected from peripheral veins using a sterile needle following sterile precautions and a volume of 1 to $2 \mathrm{ml}$ was taken in a blood culture bottle containing Brain Heart Infusion (10$20 \mathrm{ml}$ ) broth and incubated for one week at $37^{\circ} \mathrm{C}$ Subcultures were made on solid media (5 sheep blood agar and MacConkey agar) on alternate days and were incubated at $37^{\circ} \mathrm{C}$ for 24 hours.. The grown bacteria were identified by colony morphology, Gram stain, motility and standard biochemical tests. Antimicrobial susceptibility testing done by Kirby - Bauer disc diffusion technique per CLSI guidelines.

Gram positive organisms were tested with the following antibiotic discs. Ampicillin (10mcg), Ofloxacin (5mcg), Cotrimoxazole (25mcg), Gentamicin(10mcg), Erythro mycin (5mcg), Cefoxitin (30 mcg), Ceftriaxone (30mcg), Vancomycin (30 mcg) Novobiocin (1 mcg) Gram negative organisms were tested with the following antibiotic discs. Piperacillin+Tazobactam $(100 / 10 \mathrm{mcg})$ Cefoperazone + Sulbactum (75/30mcg). Gentamicin (10mcg), Amikacin (30mcg), Ciprofloxacin $(5 \mathrm{mcg})$, Ofloxacin (5mcg), Cefuroxime $(30 \mathrm{mcg})$, Ceftriaxone (30mcg), Amoxyclav (20/10mcg)

\section{Results and Discussion}

Total 65 neonates of age group upto one month were included in the study. Signs and symptoms were present in 60 neonates and 5 babies had no clinical features of sepsis. Males accounted for 43 (72\%) and 17 (28\%) were females.

Considering the maternal risk factors, prolonged rupture of membranes (PROM) was present in $25(39 \%)$ mothers followed by discharge of foul smelling liquor in 14 $(21 \%)$ and more than 3 vaginal examinations in $11(17 \%), 10(15 \%)$ mothers had fever.( Figure 1).

Majority of the patients were term $42(65 \%)$ out of which 13 (31\%) were small for gestational age (SGA) and $29(69 \%)$ were appropriate for gestational age (AGA). 23 patients were preterm of which $17(74 \%)$ were SGA while $6(26 \%)$ were AGA. On the whole, $30(46 \%)$ patients were SGA and 35 (54\%) were AGA [figure 1]. Francesca Cortese et al., 2015 has shown that preterm babies especially low birth weight babies are more prone to neonatal sepsis.

The commonly seen clinical features were respiratory distress in $33(55 \%)$, refusal to feeds or poor feeding in $29(48 \%)$, lethargy in $23(38 \%)$, jaundice in 22 (37\%), abdominal distension in $16(27 \%)$, vomiting in $16(27 \%)$, hyperthermia in $13(22 \%)$, convulsions in $10(17 \%)$, hypotension and oliguria in each of $3(5 \%)$ and sclerema in 2 (3\%). Many patients in the study had more than 2 clinical features at the time of presentation [figure 4]. Wynn et al., 2010 had mentioned many the clinical manifestations which are highly variable depending on the virulence of pathogens and on the mechanisms of host defense. 
Table.1 Antimicrobial susceptibility pattern for Gram positive organisms (Coagulase negative Staphylococci and Staphylococcus aureus)

\begin{tabular}{|l|l|l|}
\hline Antibiotic & Susceptible & Resistant \\
\hline Vancomycin & 100 & 0 \\
\hline Linezolid & 100 & 0 \\
\hline Cefoxitin & 90 & 10 \\
\hline Ofloxacin & 81 & 19 \\
\hline Ciprofloxacin & 5 & 95 \\
\hline Gentamycin & 43 & 57 \\
\hline Ceftriaxone & 85 & 15 \\
\hline Cotrimoxazole & 35 & 65 \\
\hline Erythromycin & 42 & 58 \\
\hline Ampicillin & 10 & 90 \\
\hline
\end{tabular}

Table.2 Antimicrobial susceptibility pattern for Gram negative organisms (Klebsiella pneuminiae, Escherichia coli, Citrobacter fruendii, Acinetobacter species)

\begin{tabular}{|l|l|l|}
\hline Antibiotic & Susceptible & Resistant \\
\hline Cefaperazone/sulbactam & 98 & 2 \\
\hline Piperacillin/tazobactam & 98 & 2 \\
\hline Ofloxacin & 94 & 6 \\
\hline Amikacin & 61 & 39 \\
\hline Ciprofloxacin & 20 & 80 \\
\hline Gentamycin & 60 & 40 \\
\hline Ceftriaxone & 68 & 32 \\
\hline Cotrimoxazole & 30 & 70 \\
\hline
\end{tabular}

Fig.1

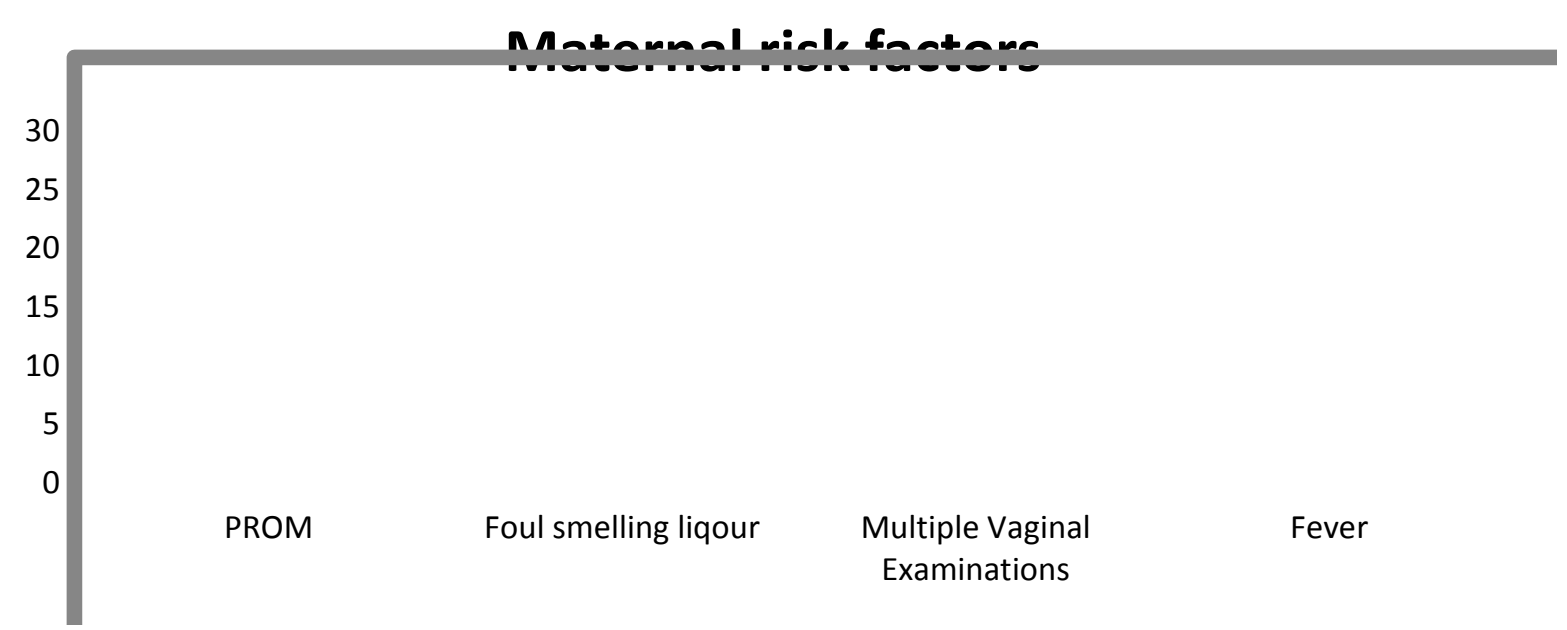




\section{ORGANISMS ISOLATED FROM UMBILCAL CORD INFECTION}
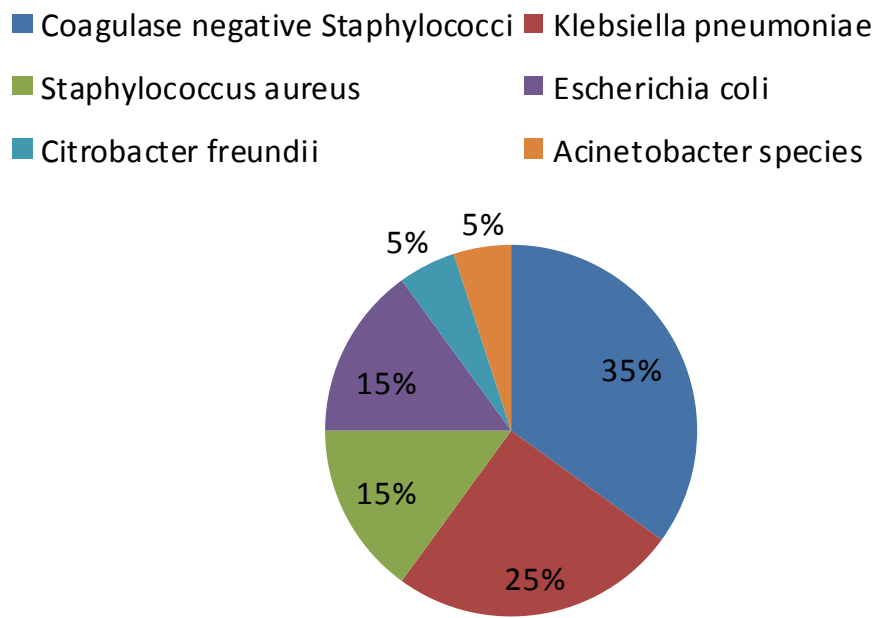

Among the babies that had omphalitis 7 (35\%) had periumbilical erythema and 13 $(65 \%)$ had umbilical discharge out of which $1(8 \%)$ had bloody discharge, $3(23 \%)$ had serosanguinous discharge and 9 (69\%) had purulent discharge. Fatima Mir et al., 2005 had a similar observation in their study.

Umbilical swab culture were positive in 20 $(94 \%)$ of the total cases. Blood culture were positive in $33(51 \%)$. Organisms grown on the umbilical swab culture were Coagulase negative staphylococci in 7 (35\%), Klebsiella pneumoniae in 5 (25\%), Escherichia coli in 3 (15\%), Staphylococcus aureus in $3(15 \%)$, Citrobacter freundii in 1 (5\%) and Acinetobacter in 1(3\%) of the omphalitis patients (Figure.2). Most common organisms isolated from blood were coagulase negative staphylococci in 14 (43\%), Klebsiella pneumoniae in 11 (33\%), Staphylococcus aureus in 5 (15\%), Citrobacter freundii in 1 (3\%) and Escherichia coli in 1 (3\%). Candida spp. was present in $1(3 \%)$ case. The results were in concordance with Vanisree et al., 2014 where Coagulase negative Staphylococci were the commonest organisms causing umbilical stump infection followed by Gram negative bacilli.

In 6 cases of septicemia, 3 cases with Klebsiella pneumoniae and 3 cases with Coagulase negative staphylococci, the same organisms were isolated in umbilical pus and blood suggesting that umbilical colonization had led to septicemia.

The Gram positive organisms showed 100\% susceptibility to Linezolid, Vancomycin, $85 \%$ to Ceftriaxone, $81 \%$ to Ofloxacin, $43 \%$ to Gentamycin and resistant to Ciprofloxacin, Cotrimoxazole Erythromycin and Ampicillin (Table 1).

Gram negative organisms were mostly susceptible to Cefaperazone/ sulbactam (98\%), Piperacillin/ Tazobactam (98\%), 
Ofloxacin (94\%) and Amikacin (61\%) and resistant to Ceftriaxone Ciprofloxacin and Gentamycin (Table:2)

In conclusion, the clinical manifestations of neonatal sepsis are highly variable and nonspecific. Umbilical stump infections may progress to neonatal sepsis. Early and correct diagnosis of the infecting organism prompt antibiotic therapy, preventive measures and supportive care will help in reducing neonatal mortality rate.

\section{References}

Bahl, R., Martines, J., Ali, N., Bhan, M.K., Carlo, W., Chan, K.Y., Darmstadt, G.L., Hamer, D.H., Lawn, J.E., McMillan, D.D. 2009. Research priorities to reduce global mortality from newborn infections by 2015. Pediatr. Infect. Dis. J., 28: S43.

Camacho-Gonzalez, A., Spearman, P.W., Stoll, B.J. 2013. Neonatal infectious diseases: evaluation of neonatal sepsis. Pediatr. Clin. North Am., 60: 367e89.

Elizabeth, A., Marchant, Guilaine, K., Boyce, Manish Sadarangani, and Pascal, M., Lavoie. 2013. Neonatal Sepsis due to Coagulase-Negative Staphylococci. Clin. Develop. Immunol., Volume (2013) Article ID 586076, 10 pages.

Fatima, Mir, Shiyam Sundar Tikmani, Sadia Shakoor, Haider Javed Warraich, Shazia Sultana, Syed Asad Ali, Anita K.M. Zaidi. 2011. Incidence and etiology of omphalitis in Pakistan: a communitybased cohort study. J. Infect. Dev. Ctries., 5(12): 828-833.
Fikree, F.F., Ali, T.S., Durocher, J.M., Rahbar, M.H. 2005. Newborn care practices in low socioeconomic settlements of Karachi, Pakistan. Soc. Sci. Med., 60: 911-921.

Francesca Cortese Pietro Scicchitano Michele Gesualdo a,Antonella Filaninno Elsa De Giorgi Federico Schettini Nicola Laforgia Marco Matteo Ciccone. 2015. Early and Late Infections in Newborns: Where Do We Stand? Pediatrics and Neonatol., 1e9.

Liu, L., H.L. Johnson, S. Cousens. et al., 2012. "Global, regional, and national causes of child mortality: an updated systematic analysis for 2010 with time trends since 2000," The Lancet, vol. 379, no. 9832, pp. 2151-2161.

Mukhopadhyay, S., Puopolo, K.M. 2012. Risk assessment in neonatal early onset sepsis. Semin Perinatol., 36: 408e15.

Mullany, L.C., Darmstadt, G.L., Katz, J., Khatry, S.K., LeClerq, S.C., Adhikari, R.K., Tielsch, J.M. 2009. Risk of mortality subsequent to umbilical cord infection among newborns of southern Nepal: cord infection and mortality. Pediatr. Infect. Dis. J., 28: 17.

Vanisree, R., Kavita Lata, M., Neelim. 2014. Uumbilical stump infections in neonates with special Reference to MRSA. World J. Pharm. Pharma. Sci., Volume 3, Issue 7, 724-731.

Wynn, J., Cornell, T.T., Wong, H.R., Shanley, T.P., Wheeler, D.S. 2010. Thehost response to sepsis and developmental impact. Pediatr., 125: $1031 \mathrm{e} 41$.

\section{How to cite this article:}

Rajesh, S., R. Vidhya Rani and Sundararajan, T. 2016. Microbiological Profile of Neonatal Sepsis with Special Reference to Umbilical Stump Infections. Int.J.Curr.Microbiol.App.Sci. 5(8): 785-790. doi: http://dx.doi.org/10.20546/ijcmas.2016.508.087 